\title{
Soluble ST2 Does Not Regulate TNF- $\alpha$ and IL-6 Production in Dengue Virus-Infected Human Monocytes
}

\author{
Marisol Pérez-Acosta, Félix Giovanni Delgado, and Jaime E. Castellanos \\ Instituto de Virología, Universidad El Bosque, Carrera 7B Bis No. 132-11, Bloque D, Bogotá 110121, Colombia \\ Correspondence should be addressed to Jaime E. Castellanos; castellanosjaime@unbosque.edu.co
}

Received 25 April 2013; Accepted 28 May 2013

Academic Editors: F. Fouque, A. Talvani, and C. Wrenger

Copyright ( 2013 Marisol Pérez-Acosta et al. This is an open access article distributed under the Creative Commons Attribution License, which permits unrestricted use, distribution, and reproduction in any medium, provided the original work is properly cited.

Dengue virus (DENV) produces an acute infection that results in the overproduction of proinflammatory cytokines. Although increased levels of the immunoregulator soluble ST2 (sST2) protein have been reported in the serum of patients with dengue, its importance during DENV infection remains unclear. The purpose of this study was to evaluate the effect of a recombinant human sST2 protein on the production of TNF- $\alpha$ and IL-6 in an in vitro model of DENV infection. Peripheral blood mononuclear cells (PBMCs) were permissive to in vitro DENV infection since viral antigen was detected in CD14+ monocytes by flow cytometry (median, 1\%; range, 0-2.2), and in their supernatants TNF- $\alpha$ and IL-6 were detected. However, sST2 protein was not detected. Using multiple staining on infected PBMC we found that only CD14+ cells produced TNF- $\alpha$ and IL-6. Treatment with human recombinant sST2 protein decreased lipopolysaccharide-induced monocyte TNF- $\alpha$ and IL- 6 production. However, this effect was not observed when the monocytes were pretreated with sST2 and later infected with DENV-2. These results suggest that sST2 has different roles in the regulation of TNF- $\alpha$ and IL- 6 expression in human monocytes stimulated with LPS and DENV-2.

\section{Introduction}

Currently, dengue is the most important arboviral disease worldwide in terms of morbidity, mortality, and economic impact [1]. Peripheral blood mononuclear cells (PBMCs) are cellular targets for dengue virus (DENV) infection [2], with monocytes being preferentially infected $[3,4]$. However, data exist suggesting that $\mathrm{T}$ and $\mathrm{B}$ lymphocytes can also become infected $[5,6]$. Following dengue infection, the virus induces production and liberation of proinflammatory cytokines such as TNF- $\alpha$ and IL- 6 , which induce changes in vascular permeability, a dominant clinical trait of severe dengue infections $[5,7-9]$.

Increased serum levels of SST2 protein have been reported in patients with dengue $[10,11]$. The interleukin-1 receptorlike-1 protein (IL1RL1), also known as ST2, is a member of the IL1R/Toll-like receptor (TLR) superfamily [12]. The ST2 gene has three isoforms generated from alternative mRNA splicing: a membrane-anchored long form (ST2L), a secreted soluble form (sST2), and a membrane-anchored variant form $(\mathrm{ST} 2 \mathrm{~V})[13,14]$. It has been postulated that both ST2L and
sST2 are involved in the control of cytokine expression during inflammatory events to regulate exacerbated inflammatory responses [15]. Treatment with recombinant sST2 protein decreases the in vitro production of proinflammatory cytokines in murine macrophages stimulated with LPS and in vivo in murine models of sepsis, arthritis, and ischemia [16-19], conditions that involve uncontrolled inflammatory reactions. The biological importance of increased serum levels of sST2 in patients with dengue remains unknown; further, the cellular source of sST2 remains undefined. Therefore, the objective of this study was to evaluate the possible immunoregulatory effects of recombinant sST2 protein on the production of proinflammatory cytokines in an in vitro model of DENV-2 infection.

\section{Materials and Methods}

2.1. Virus. DENV serotype 2 (DENV-2) was kindly supplied by the Colombian National Institute of Health and harvested using C6/36 cells. Additionally, a cellular lysate of C6/36 cells was prepared (mock inoculum) under the same conditions 
without inoculation of the virus. Viral titres were obtained by enzymatic immunodetection of foci of infected cells through monolayers of LLC-MK2 cells.

2.2. Obtaining PBMCs and Monocytes. Peripheral venous blood was collected from healthy adult donors who authorised, through means of an informed consent, the use of their blood components for this research study. This study was approved by the Ethics Committee of the Universidad El Bosque. Donors seronegative for DENV IgM and IgG (immunochromatographic kit, Dengue Duo Cassette, Panbio Diagnostics) were selected. PBMCs and monocytes were then obtained under sterile conditions using reagents with endotoxin levels under $0.3 \mathrm{EU} / \mathrm{mL}$. The PBMCs obtained through a Ficoll gradient (Hypaque-1077, Sigma) were resuspended in maintenance media (RPMI-1640, Gibco) supplemented with $10 \%$ FBS (Gibco), $100 \mathrm{U} / \mathrm{mL}$ penicillin, and $100 \mu \mathrm{g} / \mathrm{mL}$ streptomycin (Gibco). Next, cells were transferred at a density of $1 \times 10^{6}$ cells $/ \mathrm{mL}$ in $15 \mathrm{~mL}$ polypropylene tubes to avoid adherence (Becton Dickinson) or in 96-well plates (Corning) and incubated at $37^{\circ} \mathrm{C}$ in an atmosphere with $5 \% \mathrm{CO}_{2}$ and $70 \%$ humidity. Monocytes, obtained by adherence in $75 \mathrm{~cm}^{2}$ culture flasks, were detached and plated at a concentration of $2 \times 10^{4}$ cells per well in 96-well polyethylene plates.

2.3. Cell Infection with DENV-2. Infections with DENV-2 were performed at a multiplicity of infection (MOI) of 0.1. For negative controls, cells incubated with mock inoculum or cells treated with media alone were used. Depending on the assay, the PBMCs were infected for 6,12 , or 24 hours; supernatants or pelleted cells were recovered at each time point for analysis by flow cytometry.

2.4. Bioactivity of Recombinant Human sST2 (rh-sST2). Monocytes were pretreated with $10 \mu \mathrm{g} / \mathrm{mL}$ of recombinant human sST2 (rh-sST2/Fc, R\&D Systems) for 3 hours and then stimulated with $50 \mathrm{ng} / \mathrm{mL}$ of Escherichia coli lipopolysaccharide (LPS, Sigma) for 12 hours, at which time cell-free supernatants were recovered to determine the concentrations of TNF- $\alpha$ and IL- 6 using flow cytometry. For negative controls, cells treated with $10 \mu \mathrm{g} / \mathrm{mL}$ human IgG (AbD Serotec) and cells cultured with maintenance media alone were used.

2.5. Effect of rh-sST2 on Monocytes Infected with DENV-2. Monocytes were pre-treated with 10,25 , or $50 \mu \mathrm{g} / \mathrm{mL}$ of rhsST2 for 3 hours and then infected with DENV-2 at an MOI of 0.1 for 12 hours; cell-free supernatants were then recovered to determine the concentrations of TNF- $\alpha$ and IL- 6 using flow cytometry. For negative controls, cells treated with 10 , 25, or $50 \mu \mathrm{g} / \mathrm{mL}$ human IgG (AbD Serotec) and cells treated with mock inoculum were used.

2.6. Detection of Infected Cells and Intracellular Cytokines by Flow Cytometry. Dengue virus detection in cells was made using an anti-flavivirus monoclonal antibody (Chemicon, MAB8744, mouse isotype IgG2a, clone 6B6C-1), followed by a secondary anti-mouse IgG2a coupled to FITC (Becton Dickinson). The following other primary antibodies
(IgG1 isotype) were used to detect surface markers: antiCD3-Pacific Blue (Becton Dickinson), anti-CD19-APC-Cy7 (Becton Dickinson), and CD14-PerCP Cy5.5 (eBioscience). Between 10 and 12 hours before harvesting the cells for analysis, $10 \mu \mathrm{g} / \mathrm{mL}$ brefeldin A (BD GolgiPlug) was added. During staining, the cells were incubated with a viability stain (LIVE/DEAD Fixable Aqua Dead Cell Stain, Invitrogen). For intracellular staining, the cells were fixed and permeabilised with Cytofix/Citoperm solution (Becton Dickinson) according to the manufacturer's instructions. Antibodies used to detect intracellular cytokines were TNF- $\alpha$-APC (Becton Dickinson) and IL-6-PE (eBioscience). A total of 100000 events were acquired and analysed using a FACSCanto II flow cytometer (Becton Dickinson), using FACSDiva software.

2.7. Cytometric Bead Array (CBA) Assay for Cytokine Levels. The quantification of TNF- $\alpha$ and IL- 6 in culture supernatants was performed using a CBA assay (BD Biosciences) according to the manufacturer's instructions, which has a theoretical limit of detection of $0.7 \mathrm{pg} / \mathrm{mL}$ and $1.6 \mathrm{pg} / \mathrm{mL}$ for TNF- $\alpha$ and IL-6, respectively. All of the samples were acquired and analysed using a FACSCanto II flow cytometer (BectonDickinson), using FACSDiva and FCAP Array software.

2.8. Statistics. The data were analysed with the statistical program SPSS version 18. Nonparametric Mann-Whitney and Wilcoxon tests were used. In all cases, values of $P<0.05$ were considered significant.

\section{Results}

Initially, the PBMCs were infected for 24 hours at a MOI of 0.1 , and infection was verified by flow cytometry. The results showed that of the subpopulations analysed, infection was observed only in CD14+ monocytes (median, 1\%; range, 02.2) compared to CD14+ cells treated with mock inoculum (median, 0.3\%; range, 0.1-0.5) $(P<0.05)$ (Figure 1(a)). In contrast, infection was not observed in the other populations analysed (Figure 1(b)).

Simultaneously, the production of proinflammatory soluble cytokines was quantified. As shown in Figure 2, TNF$\alpha$ and IL- 6 production was detected in the supernatants from infected PBMCs at all time points assayed. However, the concentrations of TNF- $\alpha$ tended to decrease over time. At 6 hours after inoculation, the median TNF- $\alpha$ concentration was $414 \mathrm{pg} / \mathrm{mL}$ (range, 224-604); this concentration decreased to $222 \mathrm{pg} / \mathrm{mL}$ (range, 44-289) at 12 hours and to $65 \mathrm{pg} / \mathrm{mL}$ (range, 2-96) at 24 hours after inoculation (Figure 2(a)). In contrast, the concentrations of IL-6 in the culture supernatants increased over time; the median value of $5213 \mathrm{pg} / \mathrm{mL}$ (range, 3065-9381) at 6 hours after inoculation increased to $7454 \mathrm{pg} / \mathrm{mL}$ (range, 5737-12331) at 12 hours and to $11788 \mathrm{pg} / \mathrm{mL}$ (range, 8521-38509) at 24 hours after inoculation (Figure 2(b)).

Additionally, the PBMC subpopulations responsible for the production of proinflammatory cytokines were identified. CD14+ monocytes produced TNF- $\alpha$ and IL- 6 at 12 and 24 hours after infection. In contrast, neither of the two cytokines 


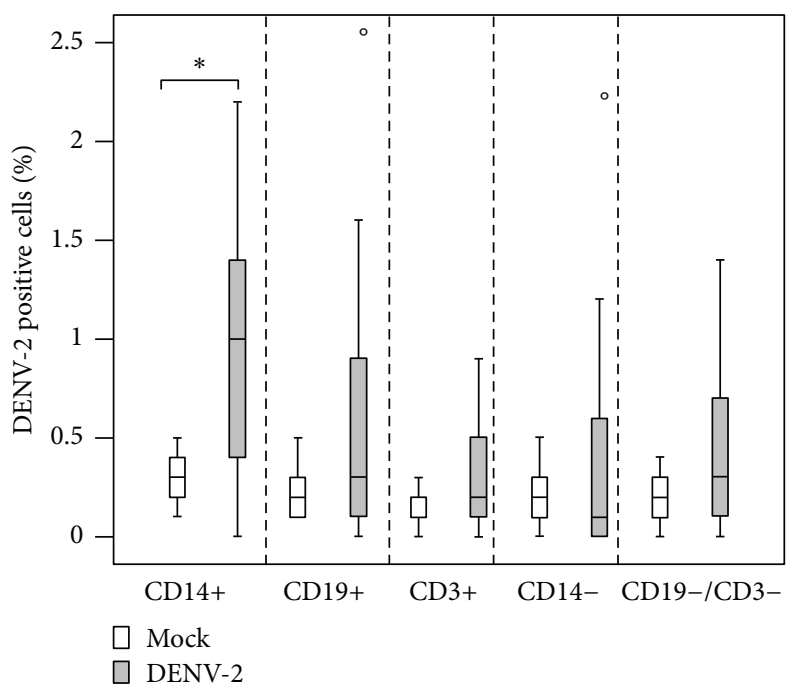

(a)
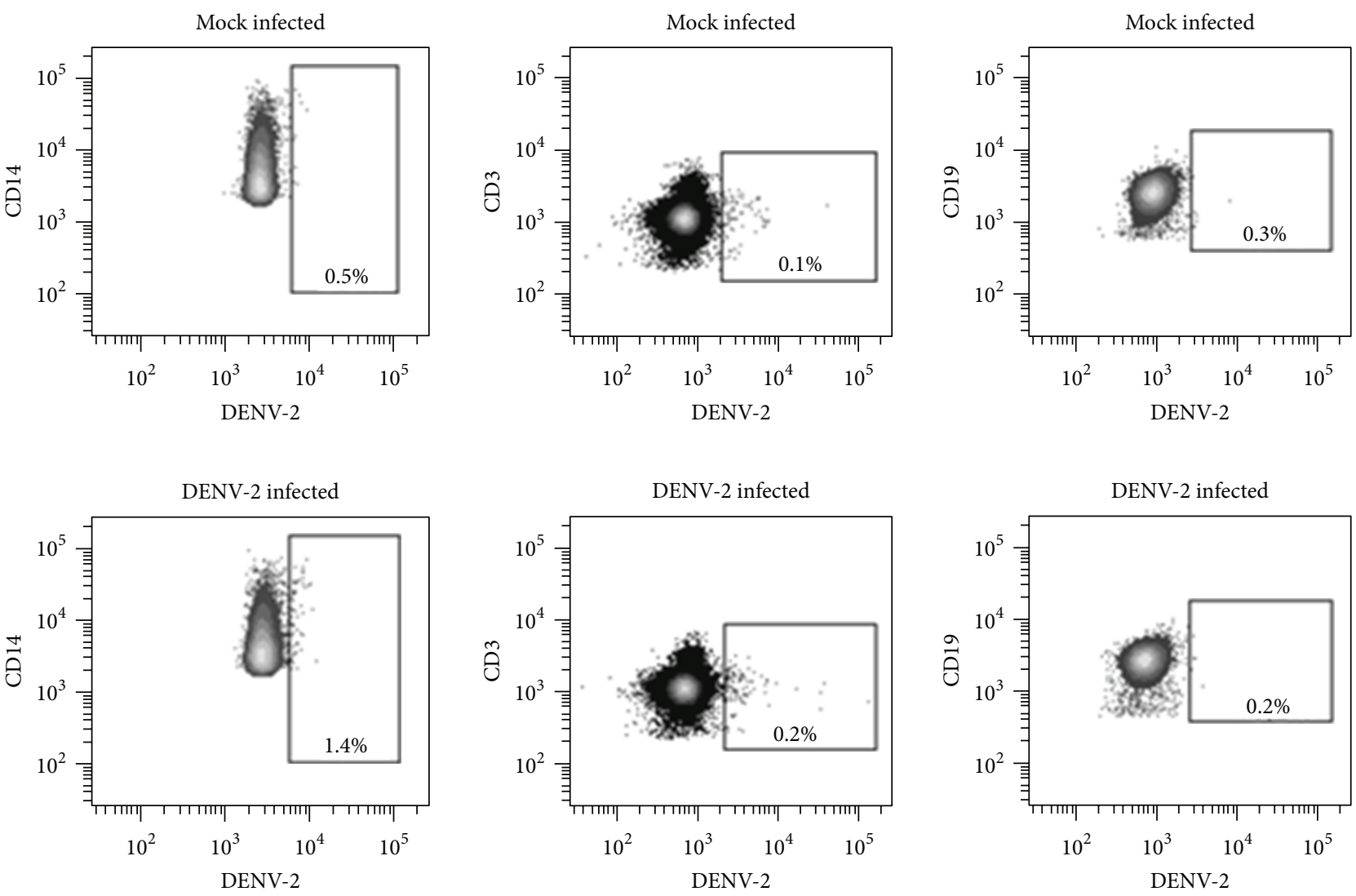

(b)

FIGURE 1: The CD14+ monocytes subpopulation is permissive to infection with DENV-2. PBMCs were infected with DENV-2 at an MOI of 0.1 for 24 hours. Significant differences were observed only in infected CD14+ monocytes compared to the mock (a). A representative result from one of the healthy subjects is shown, in whom it can be seen that only CD14+ monocytes have a higher percentage of DENV positive cells compared to mock (1.4\% versus $0.5 \%$ ) (b). Results expressed as box and whisker plots summarizing data from eight healthy subjects. Significance was calculated using the Mann-Whitney test ${ }^{*} P<0.05$. 


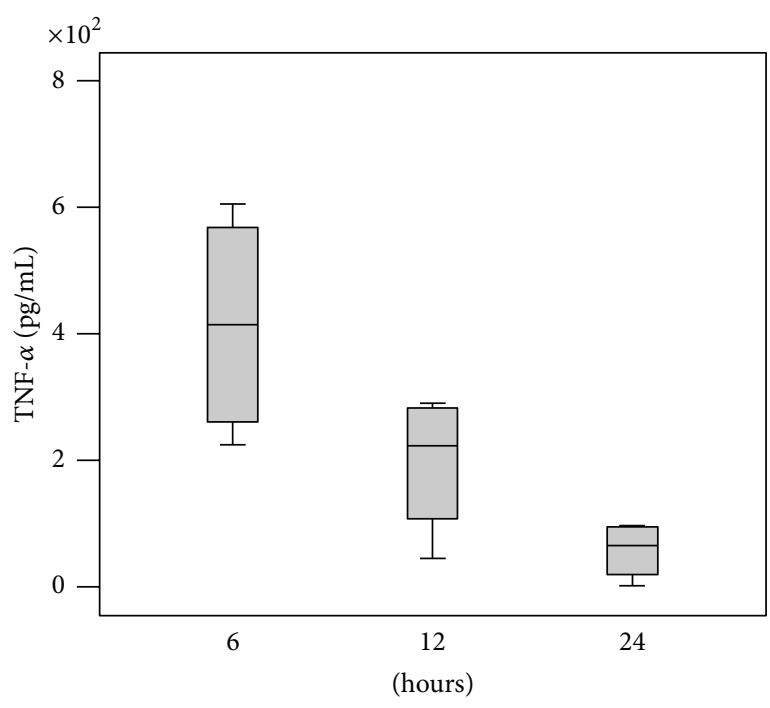

(a)

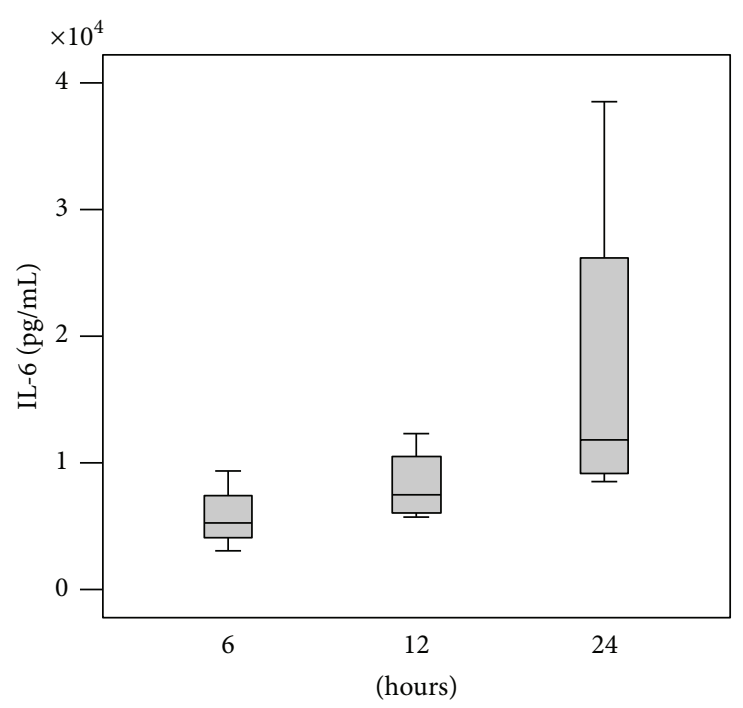

(b)

FIGURE 2: DENV-2 induces the production of proinflammatory cytokines in PBMCs. PBMCs were infected with DENV-2 at an MOI of 0.1 for 6,12, or 24 hours; concentrations of TNF- $\alpha$ (a) and IL-6 (b) were quantified in the supernatants using a CBA assay. The data were obtained after subtracting the concentration values of each cytokine quantified in the supernatants of the mock-inoculated cells. The median values obtained from mock-inoculated cells were $4 \mathrm{pg} / \mathrm{mL}$ (range, 0-14) and $125 \mathrm{pg} / \mathrm{mL}$ (range, 56-1153) for TNF- $\alpha$ and IL-6, respectively. Results expressed as box and whisker plots summarizing data from three healthy subjects.

evaluated were detected in T and B lymphocytes. As shown in Table 1, more CD14+ monocytes produced TNF- $\alpha$ and IL- 6 at 12 hours post-infection.

Previously we found that patients with acute-phase DHF/ DSS had greater sST2 levels in sera than DF patients or controls [11], but the cellular source of sST2 remains undefined. To evaluate whether PBMCs could produce sST2, an ELISA was performed to quantify the levels of sST2 in the supernatants of PBMCs infected with DENV-2 for 12, $24,48,72$, and 96 hours. sST2 was not detected in any of the four replicates from any time point for any condition, neither was sST2 detected from mock-inoculated PBMCs (data not shown). As a positive control for the ELISA, sera from patients with dengue fever or dengue hemorrhagic fever previously analyzed were used [11].

Finally, because the CD14+ monocytes were the only cellular population permissive for infection in vitro and they were responsible for the production of TNF- $\alpha$ and IL- 6 , the possible immunoregulatory effects of rh-sST2 were evaluated in this cellular population. First, the bioactivity of rh-sST2 was verified, and its effect on LPS-induced TNF- $\alpha$ and IL-6 production was evaluated. The monocytes pre-treated with rh-sST2 and LPS stimulated produced significantly less TNF- $\alpha$ and IL- 6 compared to the monocytes not pretreated with rh-sST2 and LPS stimulated (Figure 3(a)). To test whether this same effect was detectable during DENV infection, monocytes were pre-treated with different concentrations of rh-sST2 and infected. As seen in Figures 3(b) and $3(\mathrm{c})$, the median concentrations of TNF- $\alpha$ and IL- 6 in the supernatants of monocytes infected and pre-treated with $10 \mu \mathrm{g} / \mathrm{mL}$ of rh-sST2 were $854 \mathrm{pg} / \mathrm{mL}$ (range, 3521244 ) and $12374 \mathrm{pg} / \mathrm{mL}$ (range, 4479-15604), respectively.
TABLE 1: Tumor necrosis factor alpha and interleukin-6 production by human CD14+ monocytes infected with DENV.

\begin{tabular}{lccc}
\hline & \multicolumn{3}{c}{ Percentage of monocytes producing cytokine ${ }^{\mathrm{a}}$} \\
& TNF- $\alpha$ & IL-6 & TNF- $\alpha$ /IL-6 \\
\hline Mock & $0.6(0.6-0.9)$ & $0.5(0.4-0.6)$ & $0.2(0.1-0.3)$ \\
DENV-2 & & & \\
12 h p.i. & $9.9(7.9-12.5)$ & $5.6(3.4-8.5)$ & $3.6(1.8-7.2)$ \\
24 h p.i. & $0.6(0.5-1.8)$ & $2.9(0.8-28)$ & $0.8(0.7-7.0)$ \\
\hline
\end{tabular}

${ }^{\mathrm{a}}$ Percentages were expressed as the median (range) from three healthy subjects processed independently. Mock infected and DENV-infected monocytes were processed simultaneously to detect CD14+ cells and intracellular cytokine.

Contrary to what was observed with LPS, these levels were not different compared to cytokine concentrations produced by the infected monocytes that were not pre-treated with rhsST2. Equally, we did not observe significant differences when we used several concentrations (Figures 3(b) and 3(c)).

\section{Discussion}

The model of infection used in this study has characteristics that approximate the conditions of in vivo infections; in other words a lower MOI, unfractionated PBMCs, and monocytes maintained in suspension were used. Of the subpopulations analysed, only CD14+ monocytes were infected with DENV-2. Moreover, the production of TNF- $\alpha$ and IL- 6 was detected in supernatants from infected PBMCs, and CD14+ monocytes were identified as the cellular source of these cytokines during DENV-2 infection. Therefore, this is the 


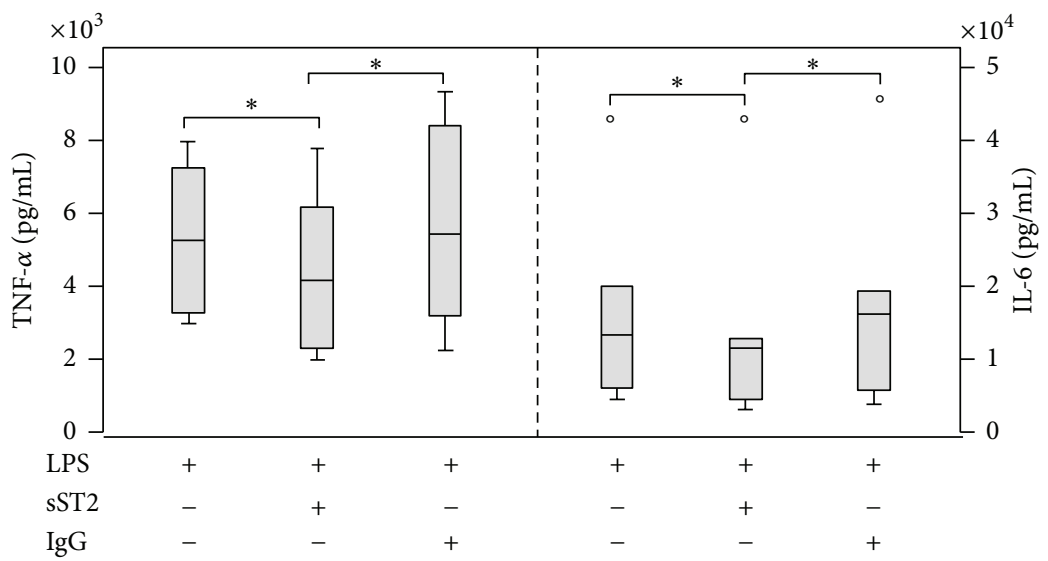

(a)

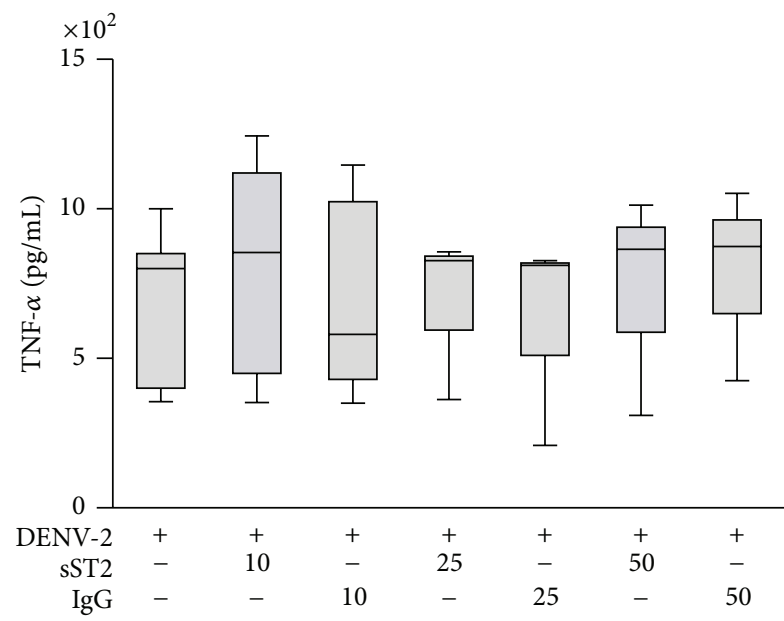

(b)

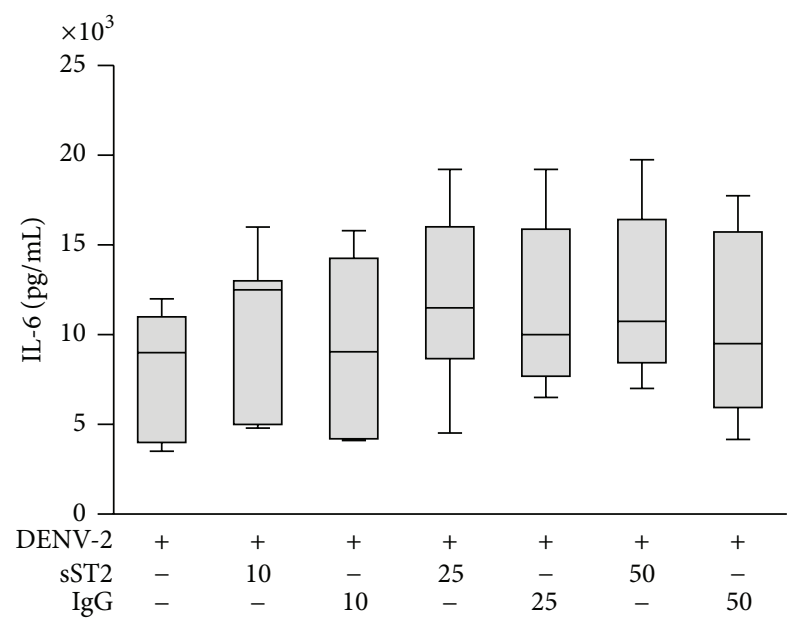

(c)

FIgURE 3: Treatment with rh-sST2 decreases the production of TNF- $\alpha$ and IL-6 in human CD14+ monocytes stimulated with LPS, but not in DENV-2-infected monocytes. Monocytes were pre-treated with $10 \mu \mathrm{g} / \mathrm{mL}$ of rh-sST2 or IgG for 3 hours and were then stimulated or not with LPS (a) or were pre-treated with 10,25 , or $50 \mu \mathrm{g} / \mathrm{mL}$ of rh-sST2 or IgG for 3 hours and were infected or not with DENV-2 using an MOI of 0.1 ((b) and (c)) for 12 hours, at which time TNF- $\alpha$ and IL-6 were quantified in the culture supernatants using a CBA assay. The data were obtained after subtracting the concentration values of each cytokine quantified in the supernatants of the mock-inoculated cells. The median values obtained from mock-inoculated cells were $25 \mathrm{pg} / \mathrm{mL}$ (range, 9-49) and $251 \mathrm{pg} / \mathrm{mL}$ (range, 202-1104) for TNF- $\alpha$ and IL-6, respectively. Results expressed as box and whisker plots summarizing data from six healthy subjects. Significance was calculated using the Wilcoxon test ${ }^{*} P<0.05$.

first study that utilised an in vitro unfractionated PBMC model of infection to simultaneously identify the cellular subtypes that were preferentially infected and responsible for the production of proinflammatory cytokines.

Previously, it has been shown that higher concentrations of sST2 are found in the dengue patients' serum [10,11]. However, the cellular source of sST2 and its role during DENV infection remain undefined. Therefore, this study aimed to address these two questions. First, sST2 was not detected in the supernatants of PBMCs infected with DENV2. These results are consistent with what has been reported in other in vitro disease model studies, where higher levels of sST2 were detected in patient serum samples. For example, in an in vitro sepsis model, Van't Veer et al. [20] was unable to detect sST2 in plasma taken from LPS-stimulated whole blood samples. Similarly, Mildner et al. [21] found that LPS stimulation of PBMC caused a massive increment of IL-1- $\alpha$, IL-1- $\beta$, IL- 6 , and TNF- $\alpha$, whereas SST2 and IL-33 were not detectable in the supernatant. In a further attempt, they found that this supernatant caused a highly significant increment of sST2 secretion, when coincubated with alveolar epithelial cells and cardiac myocytes. Wagenaar et al. [22] evaluated the in vitro production of sST2 in Leptospira-infected whole blood or PBMCs and also failed to detect sST2. Our results suggest that PBMCs are not directly responsible for the production of the sST2 that is present in the serum of patients with dengue. In contrast, there are reports demonstrating that when infected with DENV or treated with recombinant TNF- $\alpha$ or sera from dengue patients, primary human umbilical vein endothelial cell cultures (HUVECs) significantly increased both sST2 mRNA and protein expression $[11,23]$. 
Second, it has been reported that in some murine disease models, such as sepsis, arthritis, and ischemia, recombinant sST2 protein has an immunoregulatory role by suppressing the production of proinflammatory cytokines both in vitro and in vivo $[16,19]$. In this study, it is reported for the first time, using primary cell culture, that the rh-sST2 protein caused a significant reduction in LPS-induced human monocyte production of TNF- $\alpha$ and IL- 6 . However, the pretreatment with rh-sST2 did not decrease the TNF- $\alpha$ or IL6 production in monocytes infected with DENV-2.

These differences found in the response of DENVinfected or LPS-stimulated monocytes could be explained because the induction of cytokine production in monocytes can be stimulated using different signalling pathways, with sST2 only modulating one of those. Recently, it has been demonstrated that DENV is recognised by TLR-3 in U937 human monocytic cells [24], inducing the expression of IL8 through this pathway. Moreover, Brint et al. [25] demonstrated that ST2 knockout mice show an increased production of proinflammatory cytokines when IL-1R, TLR2, TLR4, and TLR9 are activated, indicating that ST2 is a negative regulator of signalling through those receptors. However, when the TLR3 receptor was stimulated, this same effect was not observed. Additionally, it has been shown that sST2 acts as a negative regulator of TLR-4 and TLR-1 signalling in murine macrophages stimulated with LPS by suppressing the mRNA expression of TLR-4 and TLR-1 $[18,19]$. However, the data are contradictory when analysing the effect of sST2 on the activation of NF- $\kappa \mathrm{B}$, a common transcription factor in the TLR signalling pathway $[18,26]$. All this evidence could suggest that the elevated levels of sST2 observed in the serum of patients with dengue could be not directly related to the regulation of the immune response during infection.

It has been suggested that the increase in sST2 observed during DENV infection is related to the disease severity. In a previous study carried out by our group [11], we found higher levels of sST2 in the serum of patients with dengue haemorrhagic fever compared to dengue fever patients. Similarly, higher levels of sST2 have been reported in the serum of patients with secondary infections, compared to patients who present a primary infection [10]. Due to the loss of vascular endothelial integrity in severe dengue infection, it is possible that there is an exposure of underlying fibroblasts to serum factors, such as TNF- $\alpha$ and IL-6, which could induce the expression of sST2 in these cells; in this way sST2 could be considered rather as a marker for tissue damage [21].

\section{Conclusions}

The results obtained in this study support the idea that CD14+ monocytes are the principal target cells for DENV and that they respond to infection by producing TNF- $\alpha$ and IL-6. Additionally, sST2 was not detected in supernatants from DENV-infected PBMCs, suggesting that this protein is not produced in vitro by PBMCs. Additionally, this study describes for the first time the immunoregulatory effect of rh-sST2 on primary cultures of human monocytes stimulated with LPS. Further, our results suggest that sST2-Fc does not exercise a direct immunoregulatory effect on the production of TNF- $\alpha$ or IL- 6 by PBMCs infected in vitro with DENV-2.

\section{Conflict of Interests}

The authors declare that no conflicting financial interests exist.

\section{Acknowledgments}

This work was supported by Universidad El Bosque and Instituto Colombiano para el Fomento de la Ciencia y la Tecnología (Colciencias, Grant 1308-493-26111).

\section{References}

[1] M. G. Guzman, S. B. Halstead, H. Artsob et al., "Dengue: a continuing global threat," Nature Reviews, vol. 8, no. 12, pp. S7S16, 2010.

[2] W.-K. Wang, T.-L. Sung, Y.-C. Tsai, C.-L. Kao, S.-M. Chang, and C.-C. King, "Detection of dengue virus replication in peripheral blood mononuclear cells from dengue virus type 2-infected patients by a reverse transcription-real-time PCR assay," Journal of Clinical Microbiology, vol. 40, no. 12, pp. 4472-4478, 2002.

[3] Z. Kou, M. Quinn, H. Chen et al., "Monocytes, but not T or $\mathrm{B}$ cells, are the principal target cells for dengue virus (DV) infection among human peripheral blood mononuclear cells," Journal of Medical Virology, vol. 80, no. 1, pp. 134-146, 2008.

[4] F. F. O. Von Sydow, M. A. Santiago, P. C. Neves-Souza et al., "Comparison of dengue infection in human mononuclear leukocytes with mosquito C6/36 and mammalian vero cells using flow cytometry to detect virus antigen," Memorias do Instituto Oswaldo Cruz, vol. 95, no. 4, pp. 483-489, 2000.

[5] Y.-W. Lin, K.-J. Wang, H.-Y. Lei et al., "Virus replication and cytokine production in dengue virus-infected human $\mathrm{B}$ lymphocytes," Journal of Virology, vol. 76, no. 23, pp. 1224212249, 2002.

[6] N. A. Mentor and I. Kurane, "Dengue virus infection of human T lymphocytes," Acta Virologica, vol. 41, no. 3, pp. 175-176, 1997.

[7] J. E. Cardier, E. Mariño, E. Romano et al., "Proinflammatory factors present in sera from patients with acute dengue infection induce activation and apoptosis of human microvascular endothelial cells: possible role of TNF- $\alpha$ in endothelial cell damage in dengue," Cytokine, vol. 30, no. 6, pp. 359-365, 2005.

[8] J. M. Carr, H. Hocking, K. Bunting et al., "Supernatants from dengue virus type- 2 infected macrophages induce permeability changes in endothelial cell monolayers," Journal of Medical Virology, vol. 69, no. 4, pp. 521-528, 2003.

[9] N. Maruo, I. Morita, M. Shirao, and S.-I. Murota, "IL-6 increases endothelial permeability in vitro," Endocrinology, vol. 131, no. 2, pp. 710-714, 1992.

[10] A. Becerra, R. V. Warke, N. de Bosch, A. L. Rothman, and I. Bosch, "Elevated levels of soluble ST2 protein in dengue virus infected patients," Cytokine, vol. 41, no. 2, pp. 114-120, 2008.

[11] N. Houghton-Triviño, D. M. Salgado, J. A. Rodríguez, I. Bosch, and J. E. Castellanos, "Levels of soluble ST2 in serum associated with severity of dengue due to tumour necrosis factor alpha stimulation," Journal of General Virology, vol. 91, no. 3, pp. 697706, 2010. 
[12] A. Dunne and L. A. J. O’Neill, “The interleukin-1 receptor/Tolllike receptor superfamily: signal transduction during inflammation and host defense," Science STKE, vol. 2003, article re3, no. 171, 2003.

[13] G. Bergers, A. Reikerstorfer, S. Braselmann, P. Graninger, and M. Busslinger, "Alternative promoter usage of the Fosresponsive gene Fit-1 generates mRNA isoforms coding for either secreted or membrane-bound proteins related to the IL-1 receptor," EMBO Journal, vol. 13, no. 5, pp. 1176-1188, 1994.

[14] H. Iwahana, K. Yanagisawa, A. Ito-Kosaka et al., "Different promoter usage and multiple transcription initiation sites of the interleukin-1 receptor-related human ST2 gene in UT-7 and TM12 cells," European Journal of Biochemistry, vol. 264, no. 2, pp. 397-406, 1999.

[15] V. Trajkovic, M. J. Sweet, and D. Xu, “T1/ST2 - an IL-1 receptorlike modulator of immune responses," Cytokine and Growth Factor Reviews, vol. 15, no. 2-3, pp. 87-95, 2004.

[16] B. P. Leung, D. Xu, S. Culshaw, I. B. McInnes, and F. Y. Liew, "A novel therapy of murine collagen-induced arthritis with soluble T1/ST2," Journal of Immunology, vol. 173, no. 1, pp. 145-150, 2004.

[17] K. Oshikawa, K. Yanagisawa, S.-I. Tominaga, and Y. Sugiyama, "ST2 protein induced by inflammatory stimuli can modulate acute lung inflammation," Biochemical and Biophysical Research Communications, vol. 299, no. 1, pp. 18-24, 2002.

[18] M. J. Sweet, B. P. Leung, D. Kang et al., "A novel pathway regulating lipopolysaccharide-induced shock by ST2/T1 via inhibition of toll-like receptor 4 expression," Journal of Immunology, vol. 166, no. 11, pp. 6633-6639, 2001.

[19] H. Yin, B.-J. Huang, H. Yang et al., "Pretreatment with soluble ST2 reduces warm hepatic ischemia/reperfusion injury," Biochemical and Biophysical Research Communications, vol. 351, no. 4, pp. 940-946, 2006.

[20] C. Van't Veer, P. S. Van Den Pangaart, M. A. D. Van Zoelen et al., "Induction of IRAK-M is associated with lipopolysaccharide tolerance in a human endotoxemia model," Journal of Immunology, vol. 179, no. 10, pp. 7110-7120, 2007.

[21] M. Mildner, A. Storka, M. Lichtenauer et al., "Primary sources and immunological prerequisites for SST2 secretion in humans," Cardiovascular Research, vol. 87, no. 4, pp. 769-777, 2010.

[22] J. F. P. Wagenaar, M. H. Gasema, M. G. A. Goris et al., "Soluble ST2 levels are associated with bleeding in patients with severe leptospirosis," PLoS Neglected Tropical Diseases, vol. 3, no. 6, article e453, 2009.

[23] R. Warke, Molecular dissection of the cellular response to dengue virus infection [Dissertation], Graduate School of Biomedical Sciences; University of Massachusetts, 2008.

[24] Y.-T. Tsai, S.-Y. Chang, C.-N. Lee, and C.-L. Kao, "Human TLR3 recognizes dengue virus and modulates viral replication in vitro," Cellular Microbiology, vol. 11, no. 4, pp. 604-615, 2009.

[25] E. K. Brint, D. Xu, H. Liu et al., "ST2 is an inhibitor of interleukin 1 receptor and Toll-like receptor 4 signaling and maintains endotoxin tolerance," Nature Immunology, vol. 5, no. 4, pp. 373$379,2004$.

[26] N. Takezako, M. Hayakawa, H. Hayakawa et al., "ST2 suppresses IL-6 production via the inhibition of I $\kappa \mathrm{B}$ degradation induced by the LPS signal in THP-1 cells," Biochemical and Biophysical Research Communications, vol. 341, no. 2, pp. 425-432, 2006. 


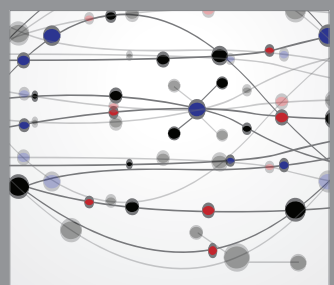

The Scientific World Journal
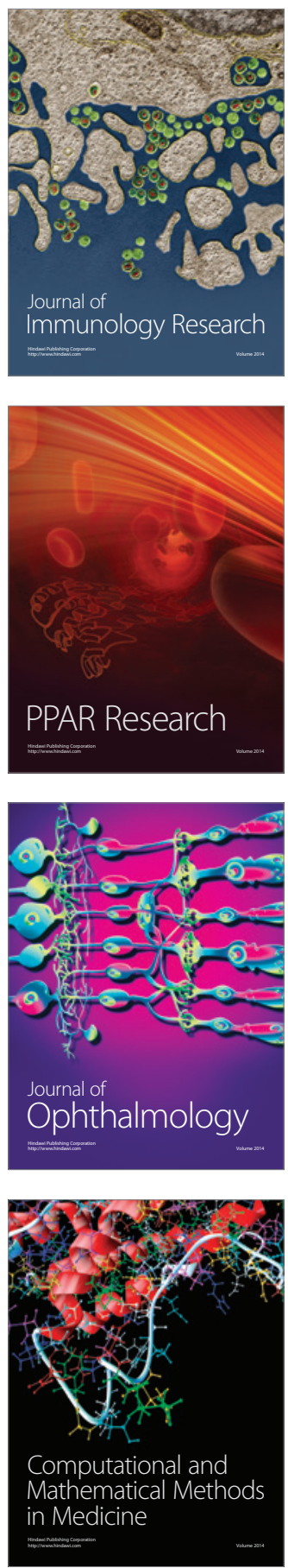

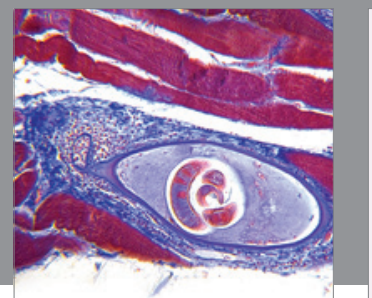

Gastroenterology

Research and Practice
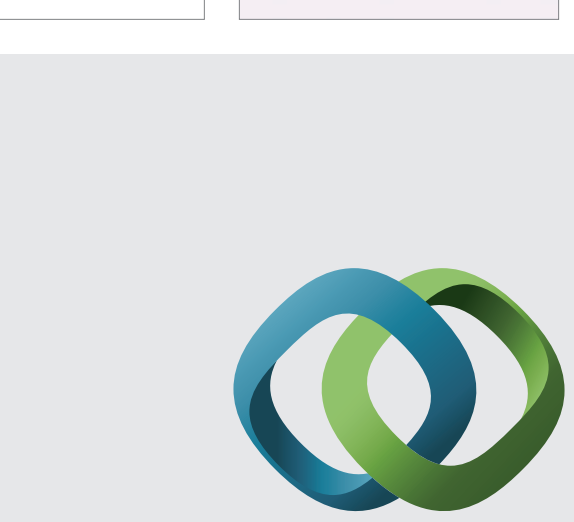

\section{Hindawi}

Submit your manuscripts at

http://www.hindawi.com
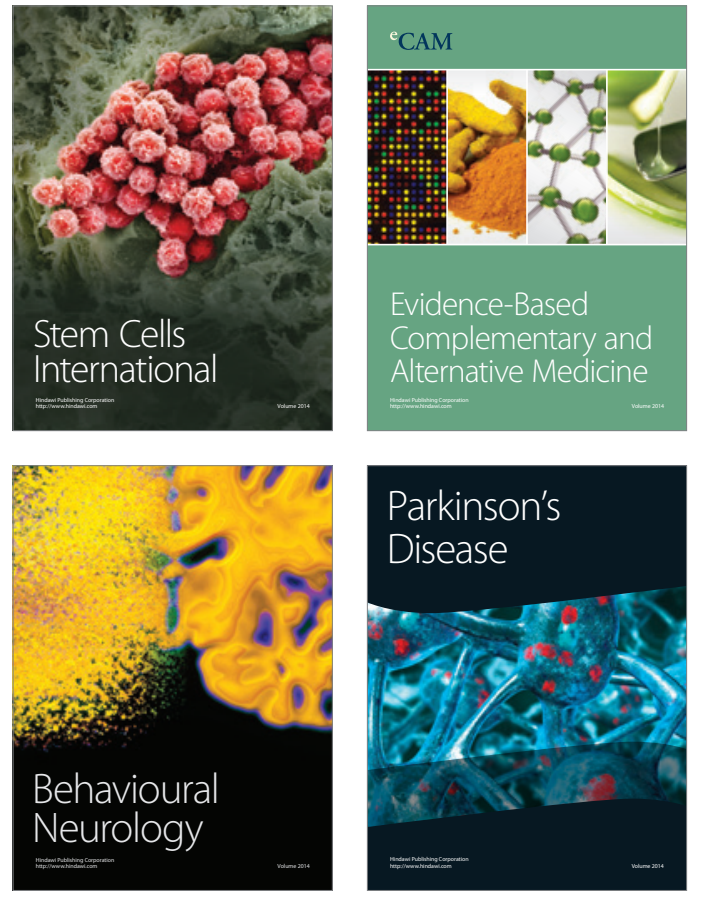
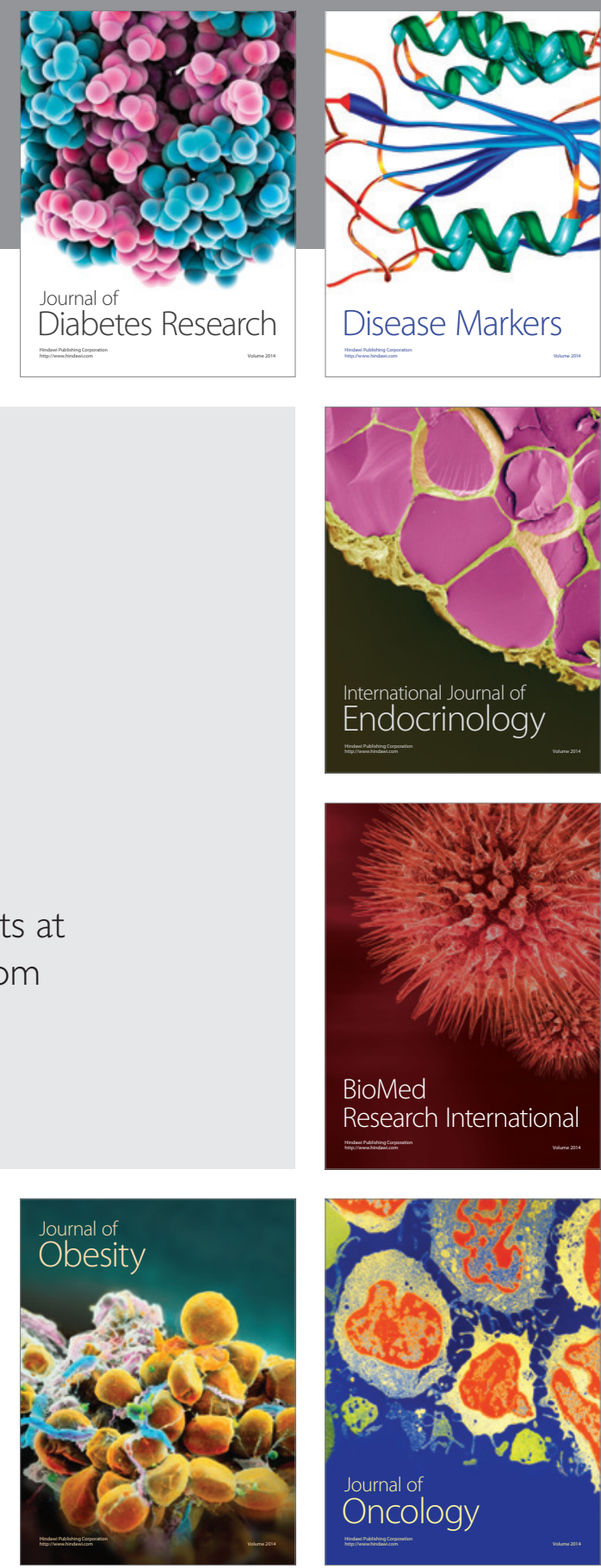

Disease Markers
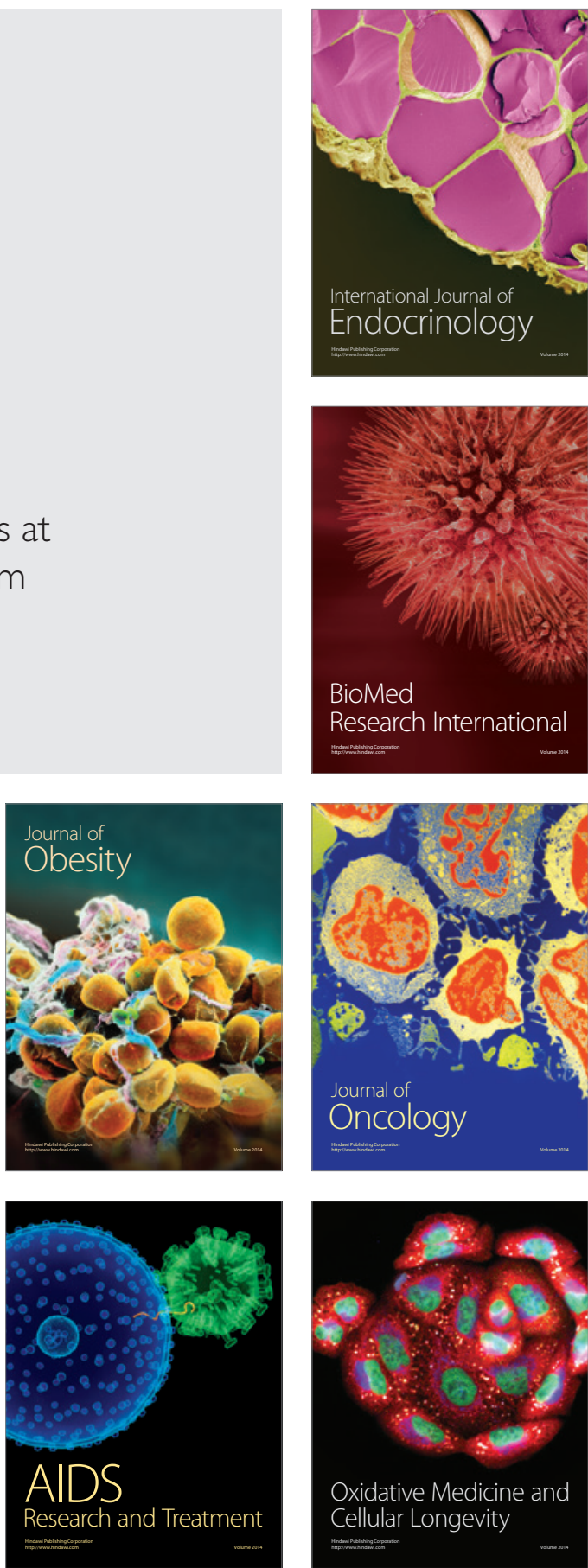